\title{
Leak Detection in Water Distribution Systems using Hydraulic Interval State Estimation
}

\author{
Stelios G. Vrachimis, Demetrios G. Eliades and Marios M. Polycarpou
}

\begin{abstract}
This work develops a new algorithm based on interval hydraulic state estimation for leakage detection in urban water distribution systems using pressure sensors and smart meters. The proposed approach takes into consideration the fact that water demand and network parameter uncertainties are unstructured, and models them as intervals defined by a corresponding upper and lower bound. The state estimation process yields bounded state estimates which are used in the creation of detection thresholds. A detection logic is used to determine whether the thresholds are violated as a result of a leakage fault event. The proposed methodology is demonstrated on a leakage benchmark dataset under various scenarios.
\end{abstract}

\section{INTRODUCTION}

Rapid climate change and increasing urban population, are growing the concern about water, one of the most precious resources in both developed and developing countries. This stresses the need to reduce the unaccounted loss of treated water in Water Distribution Systems (WDS). Such water losses can cause service disruption to the customers and an unnecessary increase to energy cost and carbon footprint. The leaked water can also damage nearby infrastructures [1]. The aging and deteriorating infrastructure of many WDS is the underlying reason of water leakages, many of which often go undetected. Leakage is a type of hydraulic fault, which may be caused by loose pipe joints and fittings, or due to cracks on the pipes which could cause a pipe to burst under the pressure of the network.

Burst event detection methods have been a subject of research for decades. Equipment-based methods, such as leak noise correlators, gas injection and pig-mounted acoustic sensing, have proven effective in detecting pipe bursts, however they are expensive, labor-intensive and may need to disrupt the service for a considerate amount of time [1] . Transient-based methods [2] attempt to detect leakages using a model-based approach, however they require measurement points with high sampling frequency and rely on a transient network model which may not be available or accurate.

Despite the advances of these methods there is still a need to further improve their reliability and efficiency, in order to detect smaller and incipient leakage faults. The latest

This work is partially funded by the European Union Horizon 2020 programme under grant agreement no. 739551 (KIOS CoE), and by the Interreg V-A Greece-Cyprus 2014-2020 programme, co-financed by the European Regional Development Fund (ERDF) and National Funds of Greece and Cyprus, under the project SmartWater2020.

S.G. Vrachimis, D.G. Eliades, M.M. Polycarpou are with the KIOS Research and Innovation Center of Excellence, Department of Electrical and Computer Engineering, University of Cyprus, Nicosia, Cyprus \{vrachimis.stelios, eldemet, mpolycar\}aucy.ac.cy developments in hydraulic sensor technology and on-line data acquisition systems have enabled water companies to deploy a larger number of more accurate pressure and flow devices with less cost. As a result, large amounts of data are now being collected and are mainly used for regulatory reporting.

These data can also be used to detect and pre-localize leaks, by applying methodologies that compare real pressure and flow data with their estimation, which is derived from a non-transient mathematical network model, as suggested by [3]. Model-based leak detection techniques based on pressure measurements have been studied in the literature [4], and consider the non-linear model of WDS, which includes several parameter and structural uncertainties. A methodology described in [5] and [6] uses a Linear Parameter Varying (LPV) model to learn the unknown model parameters and give a state estimate. Leak detection, isolation and estimation is then performed by computing residuals which are obtained by comparing measured pressures (hydraulic heads) in selected points of the network with their estimated values and zonotopes to create thresholds that consider parameter uncertainty. However, no uncertainty on water demands is considered, a factor that can make parameter identification unreliable. To maximize leakage isolability with a reasonable number of sensors, an optimal sensor placement methodology must be used, as the one described in [7].

The characterization of consumer demands is considered a critical issue for correct model calibration. In [8], the authors propose leak-detection and localization approach to be coupled with a demand calibration methodology that identifies geographically distributed parameters. The approach is based on comparing the calibrated parameters with their historical values to assess if changes in these parameters are caused by a system evolution or by the effect of leakage. However, even with a calibrated model, a statistical characterization of demands can give unreliable results. This is why researchers have proposed a more reliable modeling of demand uncertainty as intervals [9].

The contribution of this work is the development and demonstration of a new algorithm based on interval hydraulic state estimation for leakage detection in urban water distribution systems which are subject to uncertainties, by using pressure sensors and smart meters. This approach takes into consideration the fact that water demand and network parameter uncertainties are unstructured, and models them as intervals defined by upper and lower bounds [10]. The interval state estimation process gives bounded pressure state estimates which are used in the creation of thresholds. A 
detection logic is used to determine if specific residuals violate these thresholds as a result of a leakage fault event.

This paper is organized as follows: In Section II the structure of a WDS is described and the dynamic equations which are used to calculate the state of these systems are defined. Then, assuming bounded uncertainties in these equations, a hydraulic interval state estimation algorithm is developed in Section III. Using the interval state estimates, a fault detection methodology is formulated in Section IV. Finally, in Section V a case study is presented where the interval state-estimation-based fault detection methodology is applied on a benchmark network under several leakage scenarios.

\section{WATER Distribution Systems Modeling}

The topology of a WDS is modeled by a directed graph denoted as $\mathcal{G}=(\mathcal{N}, \mathcal{L})$. Let $\mathcal{N}=\left\{1, \cdots, n_{n}\right\}$ be the set of all nodes, where $|\mathcal{N}|=n_{n}$ is the total number of nodes. These represent junctions of pipes, consumer water demand locations, reservoirs and tanks. The unknown quantity associated with each node $j$ is the hydraulic head, indicated by $h_{j}$. Reservoirs are considered nodes with known head. We define the set of nodes with unknown head $\mathcal{N}_{u}=\left\{1, \cdots, n_{u}\right\}$, where $\left|\mathcal{N}_{u}\right|=n_{u}$ is the number of nodes with unknown head. The set of nodes with known head is defined as $\mathcal{N}_{h}=\left\{n_{u}+1, \cdots, n_{n}\right\}$, where $\left|\mathcal{N}_{h}\right|=n_{h}$ is the number of nodes with known head and $\mathcal{N}=\mathcal{N}_{u} \cup \mathcal{N}_{h}$. Each node $j$ with unknown head is associated with a water consumer demand at the node location, denoted by $q_{e x t, j}$.

Let $\mathcal{L}=\left\{1, \cdots, n_{l}\right\}$ be the set of links, where $|\mathcal{L}|=n_{l}$ is the total number of links. These represent network pipes, water pumps and pipe valves, with the last two being the main hydraulic control elements in a water network. The unknown quantity associated with a link $i$ is the water flow, indicated by $q_{i}$.

Each component of the WDS graph is associated with certain parameters that describe different physical properties of the network and are needed for hydraulic state estimation. For pipes, these are the roughness coefficients, length and diameter, while for nodes these are the node elevations. In this work we consider these parameters time-invariant and denote them using a single vector $\boldsymbol{\theta}$. In extension, let $\mathcal{M}(\boldsymbol{\theta} ; \mathcal{G})$ be a complete model of a WDS which associates the network graph with each network parameter.

\section{A. Hydraulics modeling}

The hydraulic state of a WDS is defined by the head at nodes and water flows in pipes and is indicated by $\boldsymbol{x}(t)=\left[\boldsymbol{h}(t)^{\top} \boldsymbol{q}(t)^{\top}\right]^{\top} \in \mathbb{R}^{n}, n=n_{u}+n_{l}$. The state is calculated using a hydraulic model of a WDS, which is a set of equations derived from the laws of: 1) conservation of energy and 2) conservation of mass in the network. In this work we use the pipe formulation of these equations as used by [11]. This formulation is also used in the widely accepted WDS simulation software EPANET [12]. The only dynamic component of these equations are the changing tank levels [13]. Using the equations of conservation of energy and mass, the hydraulic equations of a water network can be written in state-space form. A good example of the statespace formulation for WDS is presented in [14]. The statespace equations are given by:

$$
\begin{aligned}
E \underbrace{\left[\begin{array}{c}
\dot{\boldsymbol{h}}(t) \\
\dot{\boldsymbol{q}}(t)
\end{array}\right]}_{\dot{\boldsymbol{x}}(t)} & =\underbrace{\left[\begin{array}{cc}
0 & M^{\top} \\
M & G(\boldsymbol{q}(t))
\end{array}\right]}_{A(\boldsymbol{x}(t))} \underbrace{\left[\begin{array}{c}
\boldsymbol{h}(t) \\
\boldsymbol{q}(t)
\end{array}\right]}_{\boldsymbol{x}(t)}+B \boldsymbol{u}(t) \\
& +F \underbrace{\left[\begin{array}{c}
-\boldsymbol{q}_{\text {ext }}(t)-\boldsymbol{l}(t) \\
\boldsymbol{h}_{\text {ext }}
\end{array}\right]}_{\boldsymbol{d}(t)},
\end{aligned}
$$

where $E \in \mathbb{R}^{n \times n}$ identifies the dynamic states corresponding to tanks levels and multiplies them with the tank base area; $M \in \mathbb{R}^{n_{l} \times n_{u}}$ is the network incidence matrix indicating the connectivity of nodes with links (nodes with known head, such as reservoirs, are excluded from this matrix); $G(\boldsymbol{q}(t)): \mathbb{R}^{n_{l}} \mapsto \mathbb{R}^{n_{l} \times n_{l}}$, contains the nonlinear functions $g_{i}\left(q_{i} ; \boldsymbol{\theta}\right), i \in \mathcal{L}$ at its diagonal, which calculate the headloss across pipe $i$ using pipe parameters $\boldsymbol{\theta}$ and according to the Hazen-Williams empirical formula [13]; $\boldsymbol{q}_{\text {ext }}(t) \in \mathbb{R}^{n_{u}}$ are the external water demands at nodes which drive the system dynamics; $\boldsymbol{h}_{\text {ext }} \in \mathbb{R}^{n_{n}-n_{u}}$ is a vector of known heads (such as the head of reservoirs); $\boldsymbol{l}(t) \in \mathbb{R}^{n_{u}}$ is the vector of leakages which are modeled at nodes and in normal operation they are assumed to be zero. The vector $\boldsymbol{d}(t)$ is modeled as an uncontrolled, known input to the system, while matrix $F \in \mathbb{R}^{n \times n_{n}}$ identifies the demand and known head components in each equation. Note that both the demand and leakage components are not state dependent in this modeling formulation.

The control signals $\boldsymbol{u}(t) \in \mathbb{R}^{n_{c}}$ in (1) represent the ways an operator can interfere with the network operation. The matrix $B \in \mathbb{R}^{n \times n_{c}}$ identifies the control input in each equation. The modeling of control inputs here corresponds to changing the settings of pumps that exist in the network by altering the pump head-flow curve [13]. Another actuator group in a WDS are hydraulic valves on pipes. When a valve closes it changes the topology of the network, modifying the incidence matrix $M$. This kind of inputs are not modeled in this work.

\section{B. Separation of dynamic and algebraic states}

The matrix $E$ in (1) is singular due to the presence of algebraic equations, therefore it is necessary to transform the system into a non-singular form. This can be achieved by exploiting specific results from index-one singular systems, as shown in [14], [15], to divide the state vector $\boldsymbol{x}$ into two subsets: the dynamic states indicated by $\boldsymbol{x}_{1} \in \mathbb{R}^{n_{t}}$, and the algebraic states indicated by $\boldsymbol{x}_{2} \in \mathbb{R}^{\left(n-n_{t}\right)}$, where $n_{t}$ is the number of tanks (the head of tanks are the dynamic states of the system). To achieve this, the dynamic states are rearranged and separated from the algebraic states, using the similarity transformation $\boldsymbol{x}_{t}=T \boldsymbol{x}$, such that $\boldsymbol{x}_{t}=$ $\left[\begin{array}{ll}\boldsymbol{x}_{1}^{\top} & \boldsymbol{x}_{2}^{\top}\end{array}\right]^{\top}$ and produce the corresponding matrices:

$$
E_{t}=T E T^{-1}, A_{t}=T A(\boldsymbol{x}) T^{-1}, B_{t}=T B, F_{t}=T F,
$$


where $T$ is the permutation matrix that is formulated by permuting the rows of the identity matrix $I_{n \times n}$, so that the rows corresponding to tank head-states are moved to the top. Note that the tank states correspond to linear dynamic equations, therefore the nonlinearities do not change place. The transformed state-space equations can then be written as follows:

$$
\begin{aligned}
\underbrace{\left[\begin{array}{cc}
E_{11} & 0 \\
0 & 0
\end{array}\right]}_{E_{t}} \underbrace{\left[\begin{array}{c}
\dot{\boldsymbol{x}}_{1}(t) \\
\dot{\boldsymbol{x}}_{2}(t)
\end{array}\right]}_{\dot{\boldsymbol{x}}_{t}(t)}= & \underbrace{\left[\begin{array}{cc}
A_{11} & A_{12} \\
A_{21} & A_{22}\left(\boldsymbol{x}_{2}(t)\right)
\end{array}\right]}_{A_{t}\left(x_{2}(t)\right)} \underbrace{\left[\begin{array}{l}
\boldsymbol{x}_{1}(t) \\
\boldsymbol{x}_{2}(t)
\end{array}\right]}_{\boldsymbol{x}_{t}(t)} \\
& +\underbrace{\left[\begin{array}{c}
B_{1} \\
B_{2}
\end{array}\right]}_{B_{t}} \boldsymbol{u}(t)+\underbrace{\left[\begin{array}{c}
F_{1} \\
F_{2}
\end{array}\right]}_{F_{t}} \boldsymbol{d}(t),
\end{aligned}
$$

where $E_{11}, A_{11} \in \mathbb{R}^{n_{t} \times n_{t}}$, while the rest of the matrices have the appropriate size defined by the division of the states. The dynamic equations and the algebraic equations can then be written separately as follows (omitting time $(t)$ notation):

$$
\dot{\boldsymbol{x}}_{1}=\underbrace{E_{11}^{-1} A_{11}}_{A_{x_{1}}} \boldsymbol{x}_{1}+\underbrace{E_{11}^{-1} A_{12}}_{A_{x_{2}}} \boldsymbol{x}_{2}+\underbrace{E_{11}^{-1} B_{1}}_{B_{x_{1}}} \boldsymbol{u}+\underbrace{E_{11}^{-1} F_{1}}_{F_{x_{1}}} \boldsymbol{d},
$$$$
0=A_{21} \boldsymbol{x}_{1}+A_{22}\left(\boldsymbol{x}_{2}\right) \boldsymbol{x}_{2}+B_{2} \boldsymbol{u}+F_{2} \boldsymbol{d} .
$$

Notice that if we consider the algebraic states $\boldsymbol{x}_{2}$ as an input, then the dynamic equations are linear. The algebraic equations however are nonlinear and cannot be solved analytically with respect to $\boldsymbol{x}_{2}$.

\section{Sensor measurement and modeling uncertainty}

In order for state estimation of the system to be performed, measurements should be available for all nodal demand outflows. Additionally the tank levels should be either measured or estimated using the initial conditions of the system. This sensor configuration guarantees the topological observability of the network. Other sensor configurations are also possible, given that they satisfy the topological observability condition, which can be checked using the algorithm in [16].

In this work we assume a scenario where smart volume meters are installed at demand nodes. These provide the aggregated consumption of a group of consumers at predefined time steps $\Delta t_{1}$ (e.g. 2 hours). For the purpose of modelling, each aggregated consumption measurement is represented as a single node in the network graph. An average estimate of the demand outflow $\hat{\boldsymbol{d}}(t)$ in $\left(\mathrm{m}^{3} / h\right)$ at each node for the elapsed time can be calculated.

Additionally, for the purpose of fault detection, we assume pressure sensors are installed within the network at a subset of nodes $\mathcal{N}_{y} \subset \mathcal{N}$. These can communicate pressure every $\Delta t_{2}<\Delta t_{1}$ minutes (e.g. 1 hour). The sensors can be randomly placed, or a sensor placement methodology can be used off-line such as in [7] to maximize leakage fault isolability.

As it is common in WDS, the hydraulic equations are solved in discrete time depending on when the measurements arrive. Here the smallest measurement time step $\Delta t_{2}$ of the pressure measurements is used, which will be indicated by $k$. The discrete-time demand estimates $\hat{\boldsymbol{d}}(k)$ contain considerable estimation error raised from the larger time step and loss of information during the conversion of measurement units from the smart volume meter measurements. The actual demands are expressed as $\boldsymbol{d}(k)=\hat{\boldsymbol{d}}(k)+\Delta \boldsymbol{d}(k)$, where $\Delta \boldsymbol{d}(k)$ is the demand uncertainty of which the upper bound $\Delta \boldsymbol{d}^{u}(k)$ at each time step is known such that $|\Delta \boldsymbol{d}(k)|<\Delta \boldsymbol{d}^{u}(k)$. Note that the effect of leakages $l(k)$ is not considered in these bounds as they are assumed to be zero.

Model uncertainty is also present in the form of uncertain pipe parameters $\boldsymbol{\theta}$, which appear in the head-loss functions $g_{i}\left(q_{i} ; \boldsymbol{\theta}\right)$. In extension, they appear in the algebraic equations (4b). We assume that estimated pipe parameters $\hat{\theta}$ are available and are related to the true parameters $\boldsymbol{\theta}=\hat{\boldsymbol{\theta}}+\Delta \boldsymbol{\theta}$, where $\Delta \boldsymbol{\theta}$ is the parameter uncertainty of which the upper bound $\Delta \boldsymbol{\theta}^{u}$ is known such as $|\Delta \boldsymbol{\theta}|<\Delta \boldsymbol{\theta}^{u}$.

The state-space equations are then discretized using the zero-order hold method and sampling time of $\Delta t_{2}$. The discrete dynamic equations (4a) considering the aforementioned uncertainties are given below, where the discretized matrices are indicated by the superscript $d$ :

$$
\begin{gathered}
\boldsymbol{x}_{1}(k+1)=A_{x_{1}}^{d} \boldsymbol{x}_{1}(k)+A_{x_{2}}^{d} \boldsymbol{x}_{2}(k)+B_{x_{1}}^{d} \boldsymbol{u}(k) \\
+F_{x_{1}}^{d}(\hat{\boldsymbol{d}}(k)+\Delta \boldsymbol{d}(k))
\end{gathered}
$$

The algebraic equations (4b) are solved in discrete time with respect to $x_{2}$, using a nonlinear solver $f_{s}^{d}(\cdot)$, which also considers demand and pipe parameter uncertainty as follows:

$$
\boldsymbol{x}_{2}(k)=f_{s}^{d}\left(\boldsymbol{x}_{1}(k), \boldsymbol{u}(k), \hat{\boldsymbol{d}}(k)+\Delta \boldsymbol{d}(k) ; \hat{\boldsymbol{\theta}}+\Delta \boldsymbol{\theta}\right)
$$

The output of the system are the pressure measurements along with tank level measurements, and are given by:

$$
\boldsymbol{y}(k)=\left[\begin{array}{cc}
C_{1} & 0 \\
0 & C_{2}
\end{array}\right]\left[\begin{array}{l}
\boldsymbol{x}_{1}(k) \\
\boldsymbol{x}_{2}(k)
\end{array}\right]+\boldsymbol{v}(k),
$$

where $\boldsymbol{y}(k) \in \mathbb{R}^{n_{s}}$ are the pressure and tank level measurements, $n_{s}$ is the number of corresponding sensors, and $\boldsymbol{v}(k)$ is the measurement noise vector which is assumed bounded, such that $|\boldsymbol{v}(k)| \leq \boldsymbol{v}^{u}$. Moreover, we can distinguish between measurements by defining $\boldsymbol{y}_{1}(k)=C_{1} \boldsymbol{x}_{1}(k)$ as the tank level sensor measurements and $\boldsymbol{y}_{2}(k)=C_{2} \boldsymbol{x}_{2}(k)$ as the pressure sensor measurements.

\section{HYDRAULIC INTERVAL STATE ESTIMATION}

The solver described in (6), when bounds on the uncertainties are known, must be able to solve a nonlinear interval system of equations in order to calculate $\boldsymbol{x}_{2}$. The set of solutions that satisfy these equations may have a complex form that needs to be described with nonlinear functions. This is why, in the literature, 'interval solutions' are most often considered, with the aim of finding the smallest interval vector containing all the solutions. A specialized solver that finds the interval solution for such equations is the algorithm described in [10], which calculates bounds on state variables:

$$
\begin{aligned}
& {\left[\boldsymbol{x}_{2}^{l}(k), \boldsymbol{x}_{2}^{u}(k)\right]=} \\
& f_{s}^{d}\left(\boldsymbol{x}_{1}^{l}(k), \boldsymbol{x}_{1}^{u}(k), \boldsymbol{u}(k), \hat{\boldsymbol{d}}(k) ; \hat{\boldsymbol{\theta}}, \Delta \boldsymbol{\theta}^{u}, \Delta \boldsymbol{d}^{u}(k)\right),
\end{aligned}
$$




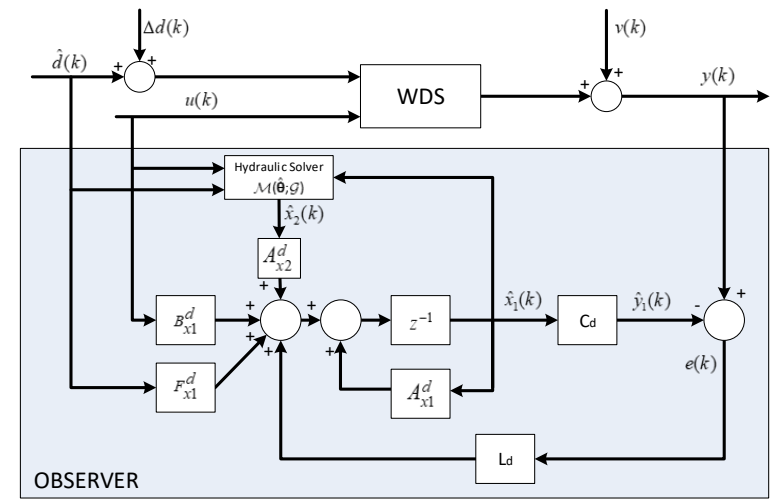

Fig. 1. Discrete time observer for tank states

where $\left[\boldsymbol{x}_{2}^{l}(k), \boldsymbol{x}_{2}^{u}(k)\right]$ defines a vector of continuous intervals, with $\boldsymbol{x}_{2}^{l}(k)$ being the lower bounds and $\boldsymbol{x}_{2}^{u}(k)$ being the upper bounds. This notation also applies to the vectors $\boldsymbol{x}_{1}^{l}, \boldsymbol{x}_{1}^{u}$ and is used throughout this work. The bounds on the pressure measurements considering also measurement noise are calculated as follows:

$$
\left[\boldsymbol{y}_{2}^{l}(k), \boldsymbol{y}_{2}^{u}(k)\right]=\left[\boldsymbol{x}_{2}^{l}(k)-\boldsymbol{v}^{u}, \boldsymbol{x}_{2}^{u}(k)+\boldsymbol{v}^{u}\right]
$$

\section{A. Observer for tank states}

The dynamic states $\boldsymbol{x}_{1}$ corresponding to tank levels may be partially measured. Assuming known initial conditions $\boldsymbol{x}_{1}(0)$ and an observable matrix pair $\left(A_{x_{1}}^{d}, C_{1}\right)$, a Luenberger linear observer can be designed that gives an estimate of $\boldsymbol{x}_{1}$. The observer uses the nonlinear hydraulic estimator of (6) to solve the algebraic states, and has the following structure which is also illustrated in Fig. 1:

$$
\begin{gathered}
\hat{\boldsymbol{x}}_{1}(k+1)=A_{x_{1}}^{d} \hat{\boldsymbol{x}}_{1}(k)+A_{x_{2}}^{d} \hat{\boldsymbol{x}}_{2}(k)+B_{x_{1}}^{d} \boldsymbol{u}(k) \\
+F_{x_{1}}^{d} \hat{\boldsymbol{d}}(k)+L_{d}\left(\boldsymbol{y}_{1}(k)-\hat{\boldsymbol{y}}_{1}(k)\right) \\
\hat{\boldsymbol{y}}_{1}(k)=C_{1} \hat{\boldsymbol{x}}_{1}(k) .
\end{gathered}
$$

The observer error dynamics are given by $\boldsymbol{e}(k+1)=$ $\left(A_{x_{1}}^{d}-L_{d} C_{1}\right) \boldsymbol{e}(k)$, where $\boldsymbol{e}(k)=\boldsymbol{y}(k)-\hat{\boldsymbol{y}}(k)$ is the error vector. The estimation error converges to zero when $\left(A_{x_{1}}^{d}-L_{d} C_{1}\right)$ has its eigenvalues inside the unit circle, thus $L_{d}$ is chosen accordingly.

\section{B. Interval observer for tank states}

Using the interval hydraulic state estimator of (8), an interval observer can be designed for the dynamic states $\boldsymbol{x}_{1}$. The interval observer consists of two sub-observers, calculating the upper and lower estimate of the state, assuming that bounds on input uncertainty and sensor noise are available. In the design of the observer the disturbance and noise are integrated in the variable they affect, resulting in that variable being bounded. The bounded estimates of the sensor measurements $\boldsymbol{y}_{1}(k)$ given by $\hat{\boldsymbol{y}}_{1}^{l}(k)$ and $\hat{\boldsymbol{y}}_{1}^{u}(k)$, converge to a lower and upper bound respectively with the condition that the system of interest is time-invariant and non-negative [17]. This is the case for the dynamic state equations of this

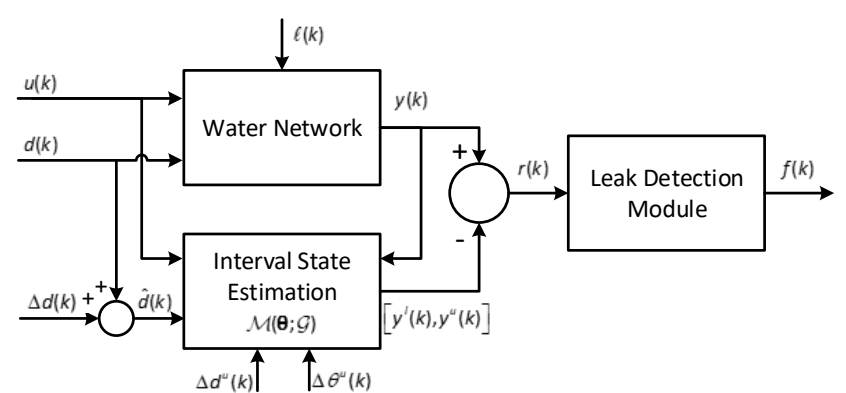

Fig. 2. Illustration of the leakage detection procedure for WDS

system. The interval observer is then given by:

$$
\begin{aligned}
\hat{\boldsymbol{x}}_{1}^{l}(k+1) & =A_{x_{1}}^{d} \hat{\boldsymbol{x}}_{1}^{l}(k)+A_{x_{2}}^{d} \hat{\boldsymbol{x}}_{2}^{l}(k)+B_{x_{1}}^{d} \boldsymbol{u}(k) \\
& +F_{x_{1}}^{d} \boldsymbol{d}^{l}(k)+L_{d}\left(\boldsymbol{y}_{1}(k)-\hat{\boldsymbol{y}}_{1}^{l}(k)\right) \\
\hat{\boldsymbol{y}}_{1}^{l}(k) & =C_{1} \hat{\boldsymbol{x}}_{1}^{l}(k)+\boldsymbol{v}^{l}(k) . \\
\hat{\boldsymbol{x}}_{1}^{u}(k+1) & =A_{x_{1}}^{d} \hat{\boldsymbol{x}}_{1}^{u}(k)+A_{x_{2}}^{d} \hat{\boldsymbol{x}}_{2}^{u}(k)+B_{x_{1}}^{d} \boldsymbol{u}(k) \\
& +F_{x_{1}}^{d} \boldsymbol{d}^{u}(k)+L_{d}\left(\boldsymbol{y}_{1}(k)-\hat{\boldsymbol{y}}_{1}^{u}(k)\right) \\
\hat{\boldsymbol{y}}_{1}^{u}(k) & =C_{1} \hat{\boldsymbol{x}}_{1}^{u}(k)+\boldsymbol{v}^{u}(k) .
\end{aligned}
$$

where $\boldsymbol{v}^{l}(k)$ and $\boldsymbol{v}^{u}(k)$ are the sensor noise vector lower and upper bound respectively, $\boldsymbol{d}^{l}(k)=\hat{\boldsymbol{d}}(k)-\Delta \boldsymbol{d}^{u}(k)$ and $\boldsymbol{d}^{u}(k)=\hat{\boldsymbol{d}}(k)+\Delta \boldsymbol{d}^{u}(k)$ are the disturbance lower and upper bounds respectively.

\section{LEAKAGE DETECTION METHODOLOGY}

Using hydraulic interval state estimation as described in Section III, we obtain bounds on the output measurement vector $\boldsymbol{y}(k)$ using uncertainty on demands and model parameters. The lower and upper bounds are given respectively by:

$$
\begin{aligned}
\boldsymbol{y}^{l}(k) & =\left[\boldsymbol{y}_{1}^{l}(k)^{\top} \boldsymbol{y}_{2}^{l}(k)^{\top}\right]^{\top} \\
\boldsymbol{y}^{u}(k) & =\left[\boldsymbol{y}_{1}^{u}(k)^{\top} \boldsymbol{y}_{2}^{u}(k)^{\top}\right]^{\top}
\end{aligned}
$$

Since the main uncertainties present in a WDS are considered in the derivation of these bounds in (13), a violation of the bounds from the sensor measurements $\boldsymbol{y}(k)$ indicates a potential fault in the system (such as a leakage). To evaluate bound violation, sensor measurements $\boldsymbol{y}(k)$ are used to calculate the residual vector as:

$$
\boldsymbol{r}(k)=\left[\begin{array}{c}
\boldsymbol{y}(k)-\boldsymbol{y}^{u}(k) \\
\boldsymbol{y}^{l}(k)-\boldsymbol{y}(k)
\end{array}\right] \in \mathbb{R}^{2 n_{s}} .
$$

The residuals are then compared with the threshold $\gamma=0$ to express the normal operation condition which is:

$$
r_{i}(k)<\gamma, \quad \forall i \in\left\{1 \cdots 2 n_{s}\right\} .
$$

A fault signature vector $\boldsymbol{f}(k) \in\{0,1\}^{2 n_{s}}$ is then defined to show threshold violations at each time step:

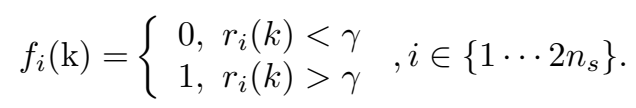

A fault is detected if there exists an $i$ for which $f_{i}(k)=1$. This procedure is illustrated in Fig. 2. 


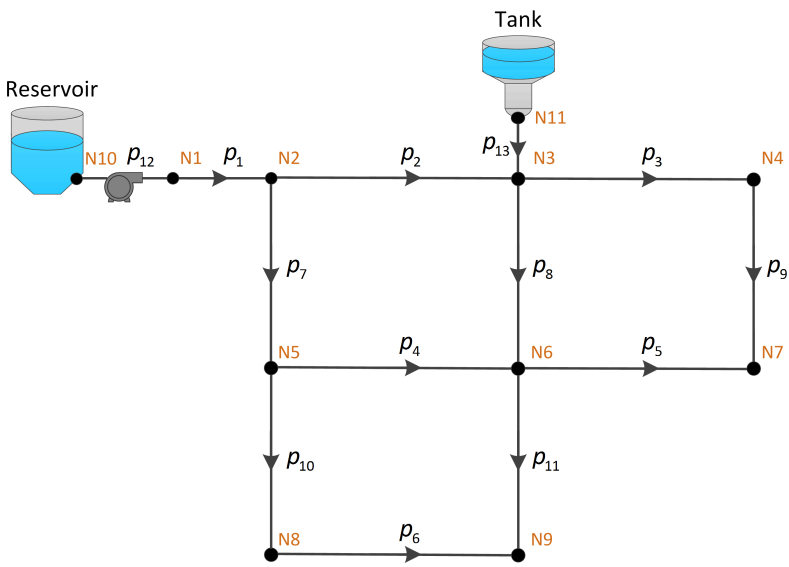

Fig. 3. The benchmark network "Net 1" on which several leakage scenarios are simulated.
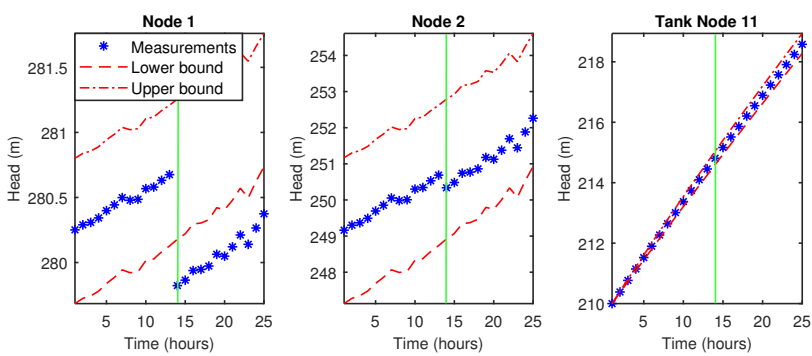

Fig. 4. Bounded state estimates for three nodes of "Net1" and the measurements from a leakage scenario, where a leakage occurs on the $14^{\text {th }}$ hour (green perpendicular line) at Node 1.

\section{CAse Study}

The benchmark network "Net1", an example network in EPANET [12] illustrated in Fig. 3, is used to demonstrate the leakage detection methodology based on interval state estimation. EPANET is a public domain, water distribution system modeling software package developed by the United States Environmental Protection Agency's Water Supply and Water Resources Division. The network model uses realistic demand patterns for a period of 24 hours. We consider known bounded uncertainties $\boldsymbol{\Delta} \boldsymbol{d}^{u}(k)$ on the estimated water demands $\hat{\boldsymbol{d}}(k)$ at each time step . Additionally, pipe parameter uncertainty is considered on the estimated pipe parameters $\hat{\boldsymbol{\theta}}$, which is also bounded by known bounds $\Delta \boldsymbol{\theta}^{u}$.

In total, 810 different scenarios are simulated in which the parameters and demands are varied randomly inside their uncertainty bounds. The demand uncertainty upper bounds $\Delta \boldsymbol{d}^{u}(k)$ and the parameter uncertainty upper bounds $\Delta \boldsymbol{\theta}^{u}$ were varied between $\pm 2 \%, \pm 5 \%$ and $\pm 10 \%$ of the estimated demands $\hat{\boldsymbol{d}}(k)$ and estimated parameters $\hat{\boldsymbol{\theta}}$ respectively. Using the defined uncertainty bounds, interval state estimation is performed as described in Section III. An example of the calculated state bounds is given in Fig. 4.

In each scenario, an abrupt leakage is induced on a node, which is random in location and magnitude. The leakages have a different profile, by varying leak hole diameter between $0 \mathrm{~cm}$ (no leak) to $4.5 \mathrm{~cm}$. The leak magnitude $l(k)$ $\left(m^{3} / h\right)$ depends on the circular hole area and the pressure at that location as follows:

$$
l(k)=c_{d} A p^{a}(k) \sqrt{\frac{2}{\rho}},
$$

where $A$ is the hole area $\left(m^{2}\right), c_{d}$ is the discharge coefficient (unitless), $p(k)(m)$ is the pressure at the node which is calculated using the head value, $a$ is the discharge coefficient, and $\rho$ is the density of the fluid (in this case, water). The simulation scenarios were generated using the Water Network Tool for Resilience (WNTR), which is an open source Python package designed to help water utilities investigate resilience of water distribution systems to hazards [18].

Measurements arrive every hour from pressure sensors installed at the nodes and a level sensor installed in the tank. Measurements have random noise with an upper bound of $\pm 2 \%$ of the measurement value. These are used for detecting leaks in the network. The level sensor measurement is also used in the dynamic tank state observer. Different sensor placement scenarios are considered, ranging from sensors covering $100 \%$ of the nodes (10 sensors) to covering $20 \%$ of the nodes ( 2 sensors). Sensor placement is performed using a greedy algorithm which prioritizes the sensor locations in which the most faults have been detected in the simulated scenarios.

The proposed detection algorithm using interval state estimation was evaluated by recording the cases in which: 1) A fault existed and was detected (True Positive TP), 2) A fault existed but was not detected (False Negative FN), 3) A fault did not exist but a fault was detected (False Positive FP), 4) A fault did not exist and was not detected (True Negative TN). The percentages of each case in relation to the number of pressure sensors in the network are presented in Table I.

\section{TABLE I}

STATISTICS FROM APPLYING THE PROPOSED FAULT DETECTION ALGORITHM TO A TOTAL OF 810 LEAKAGE SCENARIOS

\begin{tabular}{|c||c|c|c|c|}
\hline \#Sensors & TP $\%$ & FN \% & FP \% & TN \% \\
\hline 10 & 66.25 & 33.75 & 0 & 100 \\
\hline 8 & 66.25 & 33.75 & 0 & 100 \\
\hline 6 & 57.20 & 42.80 & 0 & 100 \\
\hline 4 & 55.14 & 44.86 & 0 & 100 \\
\hline 2 & 53.91 & 46.09 & 0 & 100 \\
\hline
\end{tabular}

The first observation from applying the detection methodology to this case study is that there are no false alarms $(0 \%$ False Positives and 100\% True Negatives). The explanation behind this result is the assumption that upper bounds on uncertainties are accurately known. As a result, the interval state estimation methodology produces state bounds that are guaranteed to include the true state. In this respect, the proposed methodology favors the avoidance of false alarms. The trade-off is that the conservative bounds produced by interval state estimation result in a lower percentage of True Positive detections and in accordance, there is a higher percentage of False Negatives. A less conservative selection 


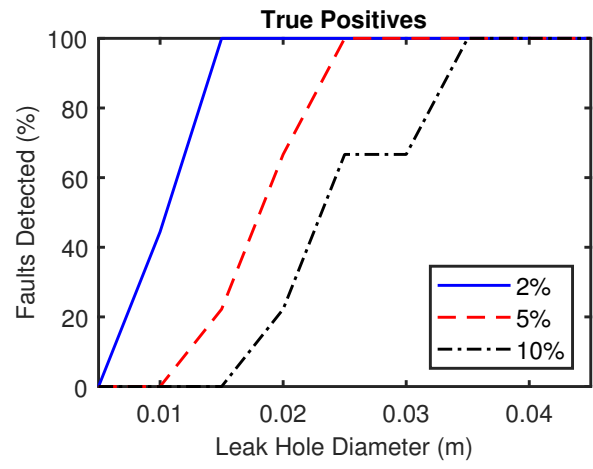

Fig. 5. Detection percentages as a function of leak diameter, for each of the three cases of upper bounds on uncertainty (10 sensors).

of the upper bounds on uncertainties would increase TP percentages, but would also introduce FP detections.

To get more insight into the results, TP percentages are plotted in Fig. 5 as a function of the leak hole diameter, for each one of the three uncertainty scenarios. The leak hole diameter is selected as the varying parameter because the leakage flow varies as a function of the node pressure, as in equation (17). Indicative leakage magnitudes are calculated by taking the average leakage flow in each simulation with the same leak hole area. This calculation reveals a flow of $2.6\left(\mathrm{~m}^{3} / \mathrm{h}\right)$ for a hole size of $5(\mathrm{~mm}), 41\left(\mathrm{~m}^{3} / \mathrm{h}\right)$ for a hole size of $2(\mathrm{~cm})$ and $158\left(\mathrm{~m}^{3} / \mathrm{h}\right)$ for a hole size of $4(\mathrm{~cm})$. As it is intuitively expected, the TP percentage increases when the leak size is increased and when the uncertainty is lower. The results presented in Fig. 5 give an indication of the required accuracy in system parameters and inputs, in order to perform leakage detection.

\section{CONCLUSION}

In this work we demonstrate an algorithm for leakage detection in urban water distribution systems based on interval hydraulic state estimation. The methodology uses pressure sensors in the network and smart meters to measure demands. Bounded demand and model parameter uncertainties are considered in the calculation of interval state estimates. The pressure sensor measurements are used to create residuals, which are in turn compared to detection thresholds created using the interval state estimates. A detection logic then determines whether the thresholds are violated as a result of a leakage fault event. A leakage benchmark dataset under various leakage, demand and uncertainty scenarios was created to test the effectiveness of the proposed methodology. The results show that the methodology is conservative as it has a relatively high percentage of undetected faults, especially when demand and modeling uncertainty are high. The detection data as a function of leakage size and uncertainty, provide insight into the required knowledge of the system inputs and parameters in order to effectively detect faults.

An extension of this work could investigate the effect on the detection statistics of choosing upper bounds on demand and modeling uncertainty that do not guarantee the inclusion of the actual values of these parameters. Additionally, an isolation module can be designed that finds the sub-graph of possible leak locations, after a leak has been detected.

\section{REFERENCES}

[1] R. Puust, Z. Kapelan, D. A. Savic, and T. Koppel, "A review of methods for leakage management in pipe networks," Urban Water Journal, vol. 7, no. 1, pp. 25-45, 2010.

[2] D. Misiunas, J. Vítkovský, G. Olsson, A. Simpson, and M. Lambert, "Pipeline break detection using pressure transient monitoring," Journal of Water Resources Planning and Management, vol. 131, no. 4, pp. 316-325, 2005.

[3] R. S. Pudar and J. A. Liggett, "Leaks in pipe networks," Journal of Hydraulic Engineering, vol. 118, no. 7, pp. 1031-1046, 1992.

[4] R. Pérez, V. Puig, J. Pascual, J. Quevedo, E. Landeros, and A. Peralta, "Methodology for leakage isolation using pressure sensitivity analysis in water distribution networks," Control Engineering Practice, vol. 19, no. 10, pp. 1157-1167, 2011.

[5] J. Vento and V. Puig, "Leak detection and isolation in pressurized water pipe networks using interval LPV models," in Networks, 2009, pp. 36-41.

[6] J. Blesa, V. Puig, J. Saludes, and J. Vento, "Leak detection, isolation and estimation in pressurized water pipe networks using LPV models and zonotopes," IFAC Proceedings Volumes, vol. 43, no. 14, pp. 36-41, 2010.

[7] M. Casillas, V. Puig, L. Garza-Castañón, and A. Rosich, "Optimal sensor placement for leak location in water distribution networks using genetic algorithms," Sensors, vol. 13, no. 11, pp. 14 984-15 005, 2013.

[8] G. Sanz, R. Pérez, Z. Kapelan, and D. Savic, "Leak detection and localization through demand components calibration," Journal of Water Resources Planning and Management, vol. 142, no. 2, p. 04015057 , 2016.

[9] D. Kang and K. Lansey, "Real-time demand estimation and confidence limit analysis for water distribution systems," Journal of Hydraulic Engineering, vol. 135, no. 10, pp. 825-837, 2009.

[10] S. G. Vrachimis, D. G. Eliades, and M. M. Polycarpou, "Real-time hydraulic interval state estimation for water transport networks: a case study," Drinking Water Engineering and Science, vol. 11, no. 1, pp. 19-24, 2018.

[11] E. Todini and S. Pilati, "A gradient algorithm for the analysis of pipe networks," in Proc. of International Conference on Computer Applications for Water Supply and Distribution, 1987.

[12] L. A. Rossman, "EPANET 2: Users manual," 2000.

[13] P. F. Boulos, K. E. Lansey, and B. W. Karney, Comprehensive Water Distribution Systems Analysis Handbook for Engineers and Planners. MWH Soft, Incorporated, 2006.

[14] F. Pasqualetti, F. Dorfler, and F. Bullo, "Attack detection and identification in cyber-physical systems," IEEE Transactions on Automatic Control, vol. 58, no. 11, pp. 2715-2729, 2013.

[15] V. L. Do, L. Fillatre, and I. Nikiforov, "A statistical method for detecting cyber/physical attacks on SCADA systems," in Proc. of Conference on Control Applications (CCA). IEEE, 2014, pp. 364369.

[16] S. Díaz, R. Mínguez, and J. González, "Topological observability analysis in water distribution systems," Journal of Water Resources Planning and Management, vol. 143, no. 5, p. 06017001, 2017.

[17] F. Mazenc, T. N. Dinh, and S. I. Niculescu, "Interval observers for discrete-time systems," International Journal of Robust and Nonlinear Control, vol. 24, no. 17, pp. 2867-2890, 2014.

[18] K. A. Klise, M. Bynum, D. Moriarty, and R. Murray, "A software framework for assessing the resilience of drinking water systems to disasters with an example earthquake case study," Environmental Modelling \& Software, vol. 95, pp. 420-431, 2017. 